\title{
Oxaloacetic Acid Supplementation as a Mimic of Calorie Restriction
}

\author{
Alan Cash*
}

Terra Biological LLC, San Diego, CA 92130, USA

\begin{abstract}
The reduction in dietary intake leads to changes in metabolism and gene expression that increase lifespan, reduce the incidence of heart disease, kidney disease, Alzheimer's disease, type-2 diabetes and cancer. While all the molecular pathways which result in extended lifespan as a result of calorie restriction are not fully understood, some of these pathways that have resulted in lifespan expansion have been identified. Three molecular pathways activated by calorie restriction are also shown to be activated by supplementing the diet with the metabolite oxaloacetic acid. Animal studies supplementing oxaloacetic acid show an increase in lifespan and other substantial health benefits including mitochondrial DNA protection, and protection of retinal, neural and pancreatic tissues. Human studies indicate a substantial reduction in fasting glucose levels and improvement in insulin resistance. Supplementation with oxaloacetic acid may be a safer method to mimic calorie restriction than the use of traditional diabetes drugs.
\end{abstract}

\section{INTRODUCTION}

For over 75 years, scientists have known how to increase average and maximal lifespan; reduce dietary intake of calories by 25 to $40 \%$ over ad libitum baseline values while maintaining adequate nutrition. This reduction in dietary intake leads to changes in metabolism and gene expression that increase lifespan, and also been reported to reduce the incidence of heart disease, kidney disease, Alzheimer's disease, type-2 diabetes and cancer in animals [1-3]. While all the molecular pathways which result in extended lifespan as a result of calorie restriction are not fully understood, some of these pathways that have resulted in lifespan expansion have been identified. Three molecular pathways identified that increase lifespan as a result of calorie restriction are 1) Activation of the energy sensor AMP protein activated Kinase (AMPK) in the presence of a functional FOXO/Daf16 transcription factor [4], 2) an increase in the Nicotinamide Adenine Dinucleotide (NAD+) to its reduced version $(\mathrm{NADH})$ within the mitochondria [5], and 3) protection of mitochondrial DNA [6]. While these molecular longevity pathways are activated within dietary restriction, reduced calorie consumption is not necessarily required to achieve the life-extension benefits conferred by activation of these beneficial molecular pathways. Dietary supplements, such as oxaloacetic acid, which activate these pathways have been shown to extent lifespan by 25\% ( $p<0.001$ ) [7]. Compounds that work to achieve some of the same benefits of the dietary restriction molecular pathways have been termed "Calorie Restriction Mimetics" [8]. This paper will discuss oxaloacetic acid supplementation, which may be used to achieve some of the health benefits of calorie restriction by mimicking the molecular longevity pathways.

\section{OXALOACETIC ACID}

Oxaloacetic acid is a dicarboxylic acid central to metabolism. It is an intermediate of both gluconeogenesis and the

*Address correspondence to this author at the Terra Biological LLC, 5033 Seachase Street, San Diego, CA 92130, USA; Tel: (858) 947-5722;

E-mail: acash@benaGene.org citric acid cycle, and is found in every cell in the body. Fig. (1) shows a schematic diagram of the citric acid cycle and the position of oxaloacetate within the cycle.

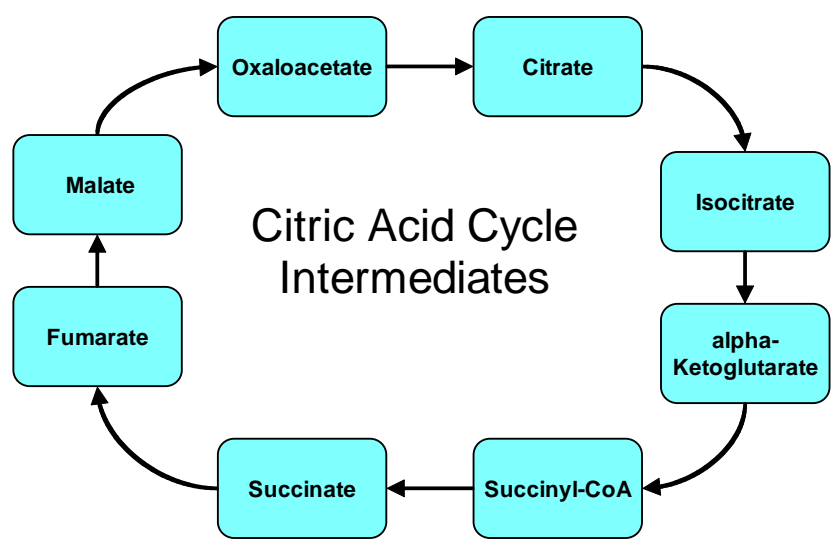

Fig. (1). Oxaloacetic acid, which in water becomes oxaloacetate, is part of the Citric Acid (Krebs) Cycle which is involved in energy production within the mitochondria. Oxaloacetic acid is a critical metabolic intermediate and is found within every mitochondrion.

The body manufactures oxaloacetate, however in some rare cases, genetic mutations in the gene that produces pyruvate carboxylase renders some individuals unable to produce enough oxaloacetate. Pyruvate carboxylase is a mitochondrial enzyme that converts pyruvate to oxaloacetate. The lack of oxaloacetate in this condition affects metabolism in at least three ways:

- The citric acid cycle is prevented from proceeding, thus limiting energy for the organism.

- Oxaloacetate is required for the first step of Gluconeogenesis, which produces fuel for the body during fasting. Certain tissues such as the heart and brain rely on the glucose produced by gluconeogenesis, and without that glucose they are severely affected. 
- $\quad$ One of the breakdown products of oxaloacetate is Aspartic acid, which is required for the urea cycle. Reduced levels of aspartic acid leads to an increase in serum ammonia levels.

The prognosis for patients with reduced levels of oxaloacetate is bleak, as progressive neurologic deterioration eventually leads to death within the first six months of life. Thus, a lack of oxaloacetate is not a preferred or healthy state [9].

Breakdown products of oxaloacetate in the body are well documented and include pyruvate, aspartic acid and malate, all of which are currently sold as, or in, dietary supplements. In nature, there are many biochemical reactions that involve oxaloacetate, which include gluconeogenesis, the glyoxylate cycle, glyoxylate degradation, mixed acid fermentation, anaerobic respiration, aerobic respiration (Krebs cycle), aspartate biosynthesis and degradation and glutamate degradation.

\section{CALORIE RESTRICTION LONGEVITY PATHWAYS STIMULATED BY OXALOACETIC ACID SUPPLE- MENTATION}

Oxaloacetic acid is a small molecule that is highly water soluble, and oral supplementation results in distribution of the compound throughout the body via the bloodstream [10]. Once in the body, oxaloacetate (the water soluble ion) can react in several ways. A highly energy favorable reaction is the conversion of oxaloacetate to L-malate, catalyzed by the enzyme malate dehydrogenase. During the conversion of oxaloacetate to malate, NADH is also converted to NAD+, which greatly increases the NAD+/NADH ratio.

When calories are reduced, the organism will produce needed glucose from pyruvate in a process called gluconeogenesis. Gene chip analysis of calorie restricted animals show a shift in metabolic gene activity that increases the activity of genes that produce the enzymes responsible

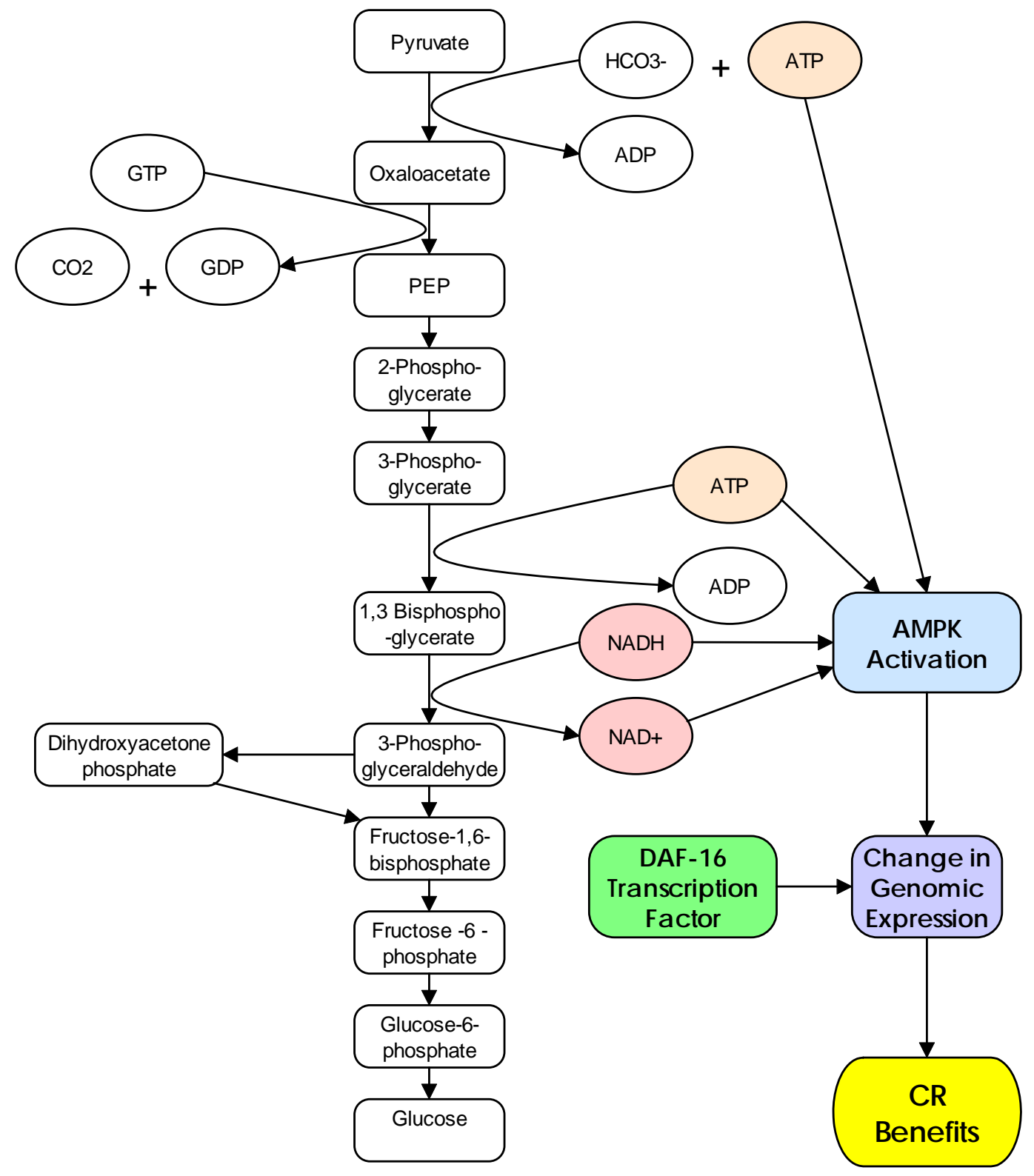

Fig. (2). Gluconeogenesis, which occurs during calorie restriction, increases the NAD+/NADH ratio, decreases ATP, and activates AMPK. With the Daf16 Transcription factor, this is thought to provide some of the benefits of the calorie restricted metabolic state. 
for gluconeogenesis [3]. As part of the gluconeogenesis process, NADH is converted to NAD+ when 1,3 bisphosphoglycerate is converted to 3-phosphoglyceraldehyde resulting in an increase in the NAD+/NADH ratio. (See Fig. 2).

An increase in the $\mathrm{NAD}^{+} / \mathrm{NADH}$ ratio has been linked to calorie restriction and increased lifespan in yeast [11, 12]. Increases in human cell lifespan have been reported by increases in nicotinamide phosphoribosyltransferase, the ratelimiting enzyme for the NAD+ salvage pathway [13], and increasing NAD+ precursors have also increased lifespan [14]. Energy levels and lifespan appear to be linked by AMPK activity, which is stimulated by increases in the $\mathrm{NAD}^{+} / \mathrm{NADH}$ ratio [15]. Activation of AMPK also appears to be the underlying mechanism of the diabetic drug metformin, which has been shown to increase lifespan in mice [16], and has been proposed as a calorie restriction mimetic. For at least one longevity pathway activated by calorie restriction, AMPK activation is required [4].

While control animals that receive oxaloacetate supplementation show a significant increase in lifespan, oxaloacetate fails to increase lifespan in animals with inactivated AMPK [7]. Thus, the activation of AMPK via an increase in the NAD+/NADH ratio appears to be a calorie restriction pathway that is mimicked by supplementation with oxaloacetate.

There may be more than one calorie restriction longevity pathway that may be stimulated by oxaloacetate supplementation. Increased mitochondrial $\mathrm{NAD}^{+}$has also been demonstrated during calorie restriction, and leads to increased cell survival during genotoxic stress [5]. A concentration of external $20 \mathrm{uM}$ oxaloacetic acid was sufficient to more than double the NAD+/NADH ratio and raise NAD+ levels by approximately $50 \%$ within the mitochondria [17]. This penetration effect of oxaloacetic acid into the mitochondria is increased by ascorbic acid (Vitamin C). The mitochondrial leakage of oxaloacetic acid across the inner mitochondrial membrane has been verified by others in more recent studies [18]. The increase in cell survival due to the increases in mitochondrial NAD+ levels may also lead to the lifespan extension seen in animals supplemented with oxaloacetate.

Mitochondrial DNA has been shown to be protected by external supplementation of oxaloacetate [19]. It is unknown if this is due to the increases in the NAD+ levels in the mitochondria, or due to the fact that oxaloacetic acid is a powerful anti-oxidant $[20,21]$. It may be that protection of the mitochondria is also a cause for the increased lifespan of animals supplemented with oxaloacetate. Calorie restriction has been shown to delay the accumulation of mitochondrial DNA deletion mutations [6]. Animal models that have abnormal mitochondrial function have shown premature aging [22], and thus enhanced maintenance of the mitochondria by oxaloacetate supplementation may lead to an increase in lifespan. (3).

Longevity pathways are shown diagrammatically in Fig.

\section{EFFECTS OF SUPPLEMENTATION WITH OX- ALOACETIC ACID}

Dietary supplementation of oxaloacetic acid has been tested in humans and animals. Yoshikawa [10] shows that 100 to $1,000 \mathrm{mg}$ of the sodium salt of oxaloacetic acid is effective in lowing the blood and urine glucose levels of diabetic patients. Yoshikawa initially investigated oxaloacetic

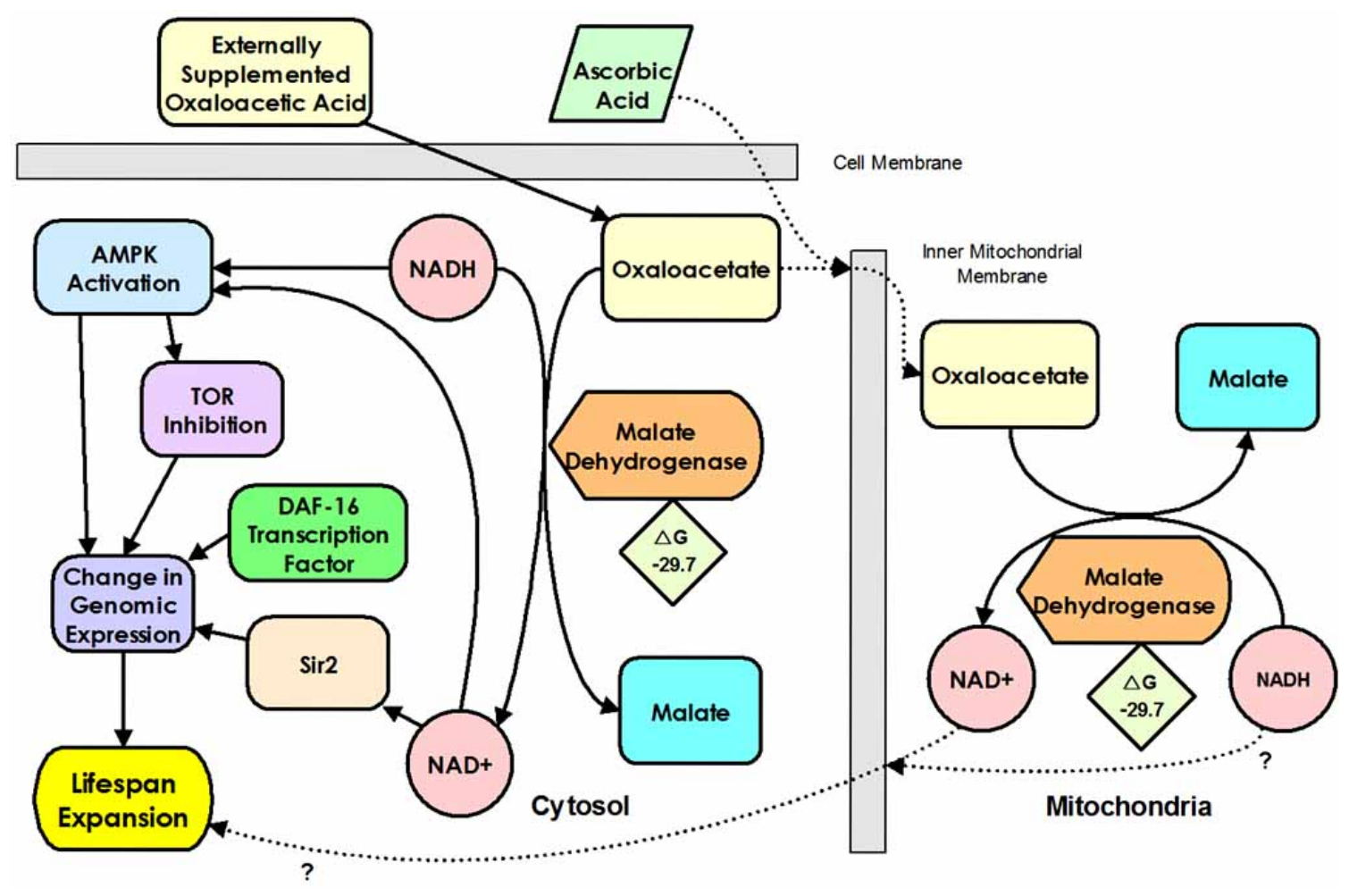

Fig. (3). Molecular pathways thought to be involved in the lifespan extension effect of oxaloacetic acid supplementation. 
acid for diabetes treatment after identifying the compound as the active ingredient extracted from the Asian mountain shrub "Euonymus alata" ("winged Euonymus", or "burning bush"), a traditional herb used for hundreds of years and still in use today to treat diabetes in Asian countries [23]. Yoshikawa showed that oral consumption of sodium oxaloacetate entered the bloodstream within one hour of consumption, and lowered fasting glucose levels in blood and urine to normal levels in the majority of diabetic patients without any noted side effects. In animal studies, Yoshikawa showed that sodium oxaloacetate increased the uptake of glucose by tissues $300 \%$ in diabetic animals, and $180 \%$ in normal animals.

The anti-diabetic activity of oxaloacetic acid is important because it may provide a safer method as a calorie restriction mimetic than other methods that include traditional diabetic drugs. The most common diabetes treatment uses the pharmaceutical metformin, which while effective, carries the risk of lactic acidosis, a potentially fatal condition. The rate of metformin induced lactic acidosis has been estimated between 3 to 8 persons/100,000 patients/year [24, 25]. Use of metformin as a calorie restriction mimetic would necessitate consumption of the drug over a lifetime. Thus, over a 20 year ingestion period from initiation, this would increase individual risk of lactic acidosis in the upper limit to over 1 in 1,000 . While this rate may be acceptable for diabetes treatment under a risk/benefit scenario, it is questionable whether this risk/benefit ratio can be supported when using metformin as a calorie restriction mimetic to achieve longer life span.

Lactic acidosis occurs when the amount of serum lactate increases faster than the systemic consumption of lactate. Lactate is produced and consumed in the following equilibrium reaction:

$$
\text { Pyruvate }+\mathrm{NADH}+\mathrm{H}^{+} \Leftrightarrow \text { Lactate }+\mathrm{NAD}^{+}
$$

The reaction is very rapid and favors Lactate. However, lactate levels can be reduced by increasing the level of $\mathrm{NAD}+$ or decreasing the NADH level (or both, resulting in an NAD+/NADH ratio increase), which will serve to reduce lactate levels and increase pyruvate production in order to maintain equilibrium. NAD+ depletion is often the rate limiting portion of the equation. Oxaloacetic acid is quite active in increasing the $\mathrm{NAD}+\mathrm{NADH}$ ratio as energetically it is preferentially converted into malate with the enzyme malate dehydrogenase. During this conversion to malate, NADH is changed to NAD+ which serves to increase the $\mathrm{NAD}+\mathrm{NADH}$ ratio. Thus, using the human metabolite oxaloacetic acid as a calorie restriction mimetic may be safer than using traditional diabetes drugs.

Calorie restriction is very effective in reducing the rate of cancer incidence [1], so it would be suspected that effective calorie restriction mimetics may have the same result. Oxaloacetic acid supplementation has been shown to eliminate the ability of some cancer cell types to reproduce, while not affecting normal tissues. $\mu \mathrm{M}$ levels of oxaloacetate in contact with human A549 lung cancer tissue in vitro resulted in differential massive debris within the cancer tissue, but not within normal tissue. The massive debris resulted in the inability of the cancer tissue to reproduce, even if the cancer tissue was moved away from the oxaloacetate solution for a period of six weeks [26]. Interestingly, the herb Euonymus alatus, the same herb from which oxaloacetic acid was extracted and identified for its anti-diabetic activity, also prevents the spread of some cancer types with low cytotoxicity. The mechanism of action appears to be inhibitory effects on matrix metalloproteinase (MMP)-9, which may be involved in tumor metastasis [27]. Halting the cellular reproduction of human lung cancer tissues with low levels of oxaloacetate in vitro is an important finding, but one that needs to be verified in vivo.

High doses of oxaloacetic acid have been shown to successfully protect mitochondrial DNA damage in brain tissues [19]. Interestingly, the oxaloacetate was delivered by intraperitoneal injection, and the protection in the brain indicates that oxaloacetate is able to penetrate the blood-brain barrier. Mitochondrial damage has been implicated in premature aging of animals [22].

In addition to protecting mitochondrial DNA, oxaloacetic acid has been shown to be protective of various whole tissues. Wood [28] showed that retinal pigmented epithelial (RPE) cells can be protected by combinations of zinc and oxaloacetate, which works much better than zinc alone. Damaged RPE cells underlie age-related macular degeneration, which causes the loss of central vision in $30 \%$ of those over 75 years old $[29,30]$.

Protection from zinc toxicity is also seen in pancreatic islet cells and neurons $[31,32]$. Protection from zinc toxicity may be due to oxaloacetate being a very powerful antioxidant. In addition to protection from zinc, oxaloacetate also provides protection of neurons from hydrogen peroxide [33] and from other free radicals [20, 21, 34, 35]. Supplementing the diet with anti-oxidants has been suggested as a method to reduce age related illness such as cardiovascular disease, the number one cause of death. Although a popular theory of anti-aging therapy involves the use of antioxidants, there is little support that anti-oxidants (in general) increase lifespan. Large epidemiologic studies of populations that ingest higher amounts of carotenoids, Vitamin $\mathrm{C}$ and Vitamin E have shown conflicting efficacy. Further, clinical trials have failed to find significant protection provided by Vitamin C, Vitamin E and Carotenoids [37]. Perhaps the unique abilities of oxaloacetic acid stem not from general anti-oxidant abilities, but from proper placement of the antioxidant into the mitochondria, via mitochondrial membrane leakage [17].

A concern in proposing oxaloacetic acid as a nutritional supplement is the effect the acid will have on the digestive system. Studies in rats, however, indicate that dicarboxylic acids such as oxaloacetic acid have a positive effect on the digestive tract, and inhibit ulceration [36]. Additionally, formal chronic and acute toxicity studies indicate a very low toxicity of oxaloacetic acid, similar to Vitamin C levels (unpublished data).

\section{MIMICKING CALORIE RESTRICTION AS A METHOD TO SUPPORT GLUCOSE METABOLISM -A CASE STUDY}

Here we report a case study on a 73 year old Hungarian woman, weight of $89 \mathrm{~kg}$, with a history of difficult to treat Type 2 diabetes. The trial was a "grass-roots" look at glucose levels performed by the patient with a glucose test strip meter under normal living conditions. The unsolicited data 
was supplied to us as the developer of the nutritional supplement product. Unfortunately, no other measurements were taken by the patient other than blood glucose levels, but the number of readings taken by the patient is impressive, and does show statistically significant results.

At the start of the study, the woman was on the following medications:

- Diaprel MR/ (80 mg)- 2 per day - Extended release Gliclazide (80 $\mathrm{mg})$, a once per day diabetic drug

- Pentoxyl-EP (400 mg) - 1 per day-- Contains pentoxifylline, used for intermittent Claudication (A symptom complex characterized by leg pain and weakness brought on by walking, with the disappearance of the symptoms following a brief rest).

- Merckformin (1,000 mg)- 1 per day- (Metformin, Glucophage) - used for Type 2 diabetes

- Glycerine sol (0.5 dl) After dinner

- $\quad$ Avandamet- 1 per day- a combination of metformin and rosiglitazone used for diabetes

Her fasting glucose levels fluctuated from the 8 to 11 $\mathrm{mmol} / \mathrm{L}$ and glucose levels after a meal increased up to 12 $\mathrm{mmol} / \mathrm{L}$.

In addition to her current medicines, the patient self started $200 \mathrm{mg} /$ day benaGene (1 capsule), an over-thecounter nutritional supplement composed of $100 \mathrm{mg} 3-$ carboxy-3-oxopropanoic acid (oxaloacetic acid) and $100 \mathrm{mg}$ ascorbic acid (Vitamin C). During the study the patient increased the use of benaGene to $400 \mathrm{mg} /$ day (two capsules), then $600 \mathrm{mg} /$ day (3 capsules).

By the end of the 70 day study the patient's fasting glucose levels had dropped to a range between 7 and 8 $\mathrm{mmol} / \mathrm{L}$, and glucose levels after a meal remained more consistent in the 7 to $8.5 \mathrm{mmol} / \mathrm{L}$ range, levels not achievable with the three prescription medications the patient was consuming. Using linear trend analysis, the patient's fasting glucose levels dropped $23 \%$ from the start of the test to the end of the test. Her glucose levels after a meal dropped $34.5 \%$, indicating a major improvement in glucose management and glucose tolerance. Comparison of the various glucose levels in the first half of the trial versus the second half of the trail yields a $\mathrm{p}$ value of $<0.001$, indicating a very significant difference between the first half and second half of the trial. The reduction in glucose levels occurred despite a reduction in the amount of Mercformin (Metformin, Glucophage) from 1,000 mg/day to $850 \mathrm{mg} /$ day during the study. The patient also stopped using Pentoxyl-EP and glycerol by the end of the study. The data from this case study is graphed as Fig. (4).

Improvement in this patient's glucose levels and glucose tolerance with the bio-identical human metabolic compound oxaloacetic acid, an over-the-counter dietary supplement, is a low-risk option that has been shown to successfully support proper glucose functioning.

\section{CONCLUSIONS}

Dietary supplementation with oxaloacetic acid can successfully mimic some of the molecular longevity pathways seen in calorically restricted animals. Animal studies show an increase in lifespan and other substantial health benefits including mitochondrial DNA protection, and protection of retinal, neural and pancreatic tissues. Human studies with oxaloactate indicate a substantial reduction in fasting glucose levels and improvement in insulin resistance. Human lung cancer cells fail to reproduce after exposure to oxaloacetic acid in culture. Oxaloacetate supplementation may be a safer method to mimic calorie restriction than the use of traditional diabetes drugs.

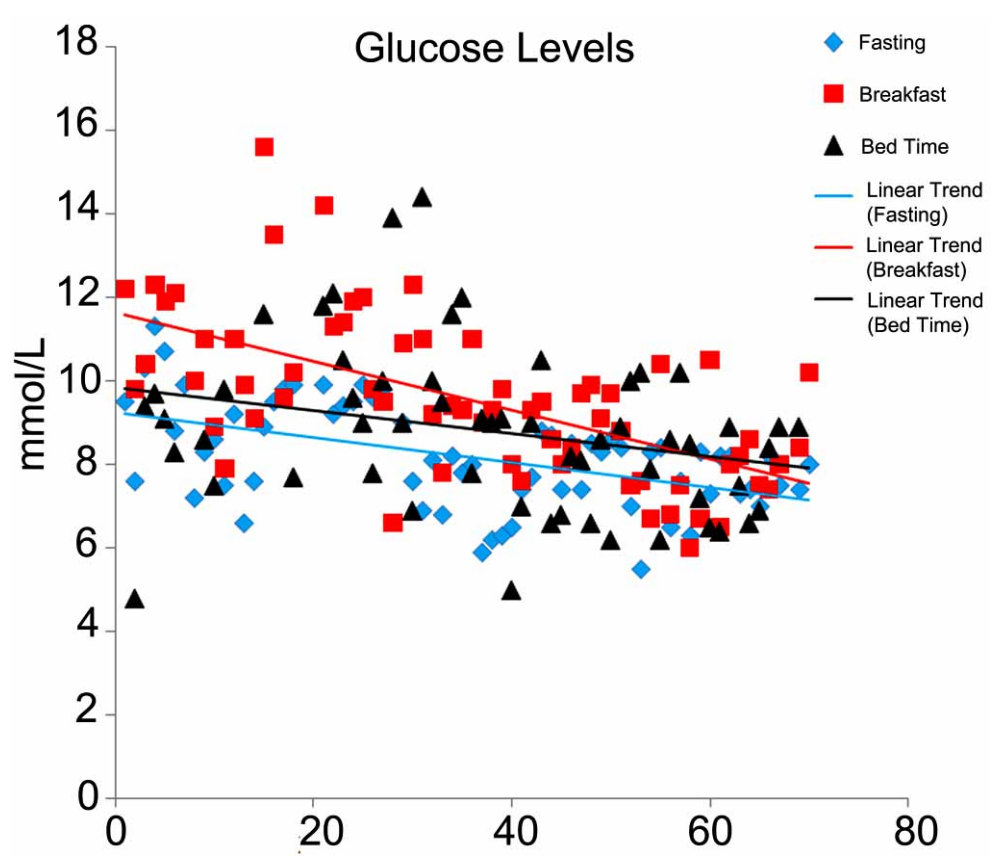

Fig. (4). Daily Blood Glucose levels from a 73 year old female diabetic patient taken under fasting conditions, after breakfast and prior to bed using an oral supplement of oxaloacetic acid. 


\section{CONFLICT OF INTEREST}

Author A. Cash is an officer in a commercial venture involving calorie restriction mimetics, including the sales of oxaloacetic acid supplemented with vitamin $\mathrm{C}$ as "benaGene"

\section{REFERENCES}

[1] Mai V, Colbert LH, Berrigan D, et al. Calorie restriction and diet composition modulate spontaneous intestinal tumorigenesis in Apc(Min) mice through different mechanisms. Cancer Res 2003; 63: $1752-5$.

[2] Koubova J, Guarente L. How does calorie restriction work? Genes Dev 2003; 17: 313-21.

[3] Dhahbi JM, Mote PL, Wingo J, Tillman JB, Walford RL, Spindler SR. Calories and aging alter gene expression for gluconeogenic, glycolytic, and nitrogen-metabolizing enzymes. Am J Physiol 1999; 277: E352-60.

[4] Greer EL, Dowlatshahi D, Banko MR, et al. An AMPK-FOXO pathway mediates longevity induced by a novel method of dietary restriction in C. elegans. Curr Biol 2007; 17: 1646-56.

[5] Yang H, Yang T, Baur JA, et al. Nutrient-sensitive mitochondrial NAD+ levels dictate cell survival. Cell 2007; 130: 1095-107.

[6] McKiernan SH, Tuen VC, Baldwin K, Wanagat J, Djamali A, Aiken JM. Adult-onset calorie restriction delays the accumulation of mitochondrial enzyme abnormalities in aging rat kidney tubular epithelial cells. Am J Physiol Renal Physiol 2007; 292: F1751-60.

[7] Williams, D.S.; Cash, A.B.; Hamadan, L.; Diemer, T. Oxaloacetate supplementation increases lifespan in $C$. elegans through an AMPK/FOXO-dependent pathway. Aging Cell 2009; Accepted Article"; doi: 10.1111/j.1474-9726.2009.00527.x.

[8] Lane MA, Ingram DK, Roth GS. The serious search for an antiaging pill. Sci Am 2002; 287: 36-41.

[9] Ahmad A, Kahler SG, Kishnani PS, et al. Treatment of pyruvate carboxylase deficiency with high doses of citrate and aspartate. Am J Med Genet 1999; 87: 331-8.

[10] Yoshikawa K. Studies on the anti-diabetic effect of sodium oxaloacetate. Tohoku J Exp Med 1968; 96: 127-41.

[11] Lin SJ, Guarente L. Nicotinamide adenine dinucleotide, a metabolic regulator of transcription, longevity and disease. Curr Opin Cell Biol 2003; 15: 241-6.

[12] Easlon E, Tsang F, Skinner C, Wang C, Lin SJ. The malateaspartate NADH shuttle components are novel metabolic longevity regulators required for calorie restriction-mediated life span extension in yeast. Genes Dev 2008; 22: 931-44.

[13] van der Veer E, Ho C, O'Neil C, et al. Extension of human cell lifespan by nicotinamide phosphoribosyltransferase. J Biol Chem 2007; 282: 10841-5.

[14] Belenky P, Racette FG, Bogan KL, McClure JM, Smith JS, Brenner C. Nicotinamide riboside promotes Sir2 silencing and extends lifespan via Nrk and Urh1/Pnp1/Meu1 pathways to NAD+. Cell 2007; 129: 473-84.

[15] Rafaeloff-Phail R, Ding L, Conner L, et al. Biochemical regulation of mammalian AMP-activated protein kinase activity by NAD and NADH. J Biol Chem 2004; 279: 52934-9.

[16] Anisimov VN, Berstein LM, Egormin PA, et al. Metformin slows down aging and extends life span of female SHR mice. Cell Cycle 2008; 7: 2769-73.

[17] Haslam JM, Krebs HA. The permeability of mitochondria to oxaloacetate and malate. Biochem J 1968; 107: 659-67.
[18] Johnson JD, Creighton DJ, Lambert MR. Stereochemistry and function of oxaloacetate keto-enol tautomerase. J Biol Chem 1986; 261: 4535-41.

[19] Yamamoto HA, Mohanan PV. Effect of alpha-ketoglutarate and oxaloacetate on brain mitochondrial DNA damage and seizures induced by kainic acid in mice. Toxicol Lett 2003; 143: 115-22.

[20] Puntel RL, Nogueira CW, Rocha JB. Krebs cycle intermediates modulate thiobarbituric acid reactive species (TBARS) production in rat brain in vitro. Neurochem Res 2005; 30: 225-35.

[21] Puntel RL, Roos DH, Grotto D, Garcia SC, Nogueira CW, Rocha JB. Antioxidant properties of Krebs cycle intermediates against malonate pro-oxidant activity in vitro: a comparative study using the colorimetric method and HPLC analysis to determine malondialdehyde in rat brain homogenates. Life Sci 2007; 81: 5162.

[22] Navarro CL, Cau P, Levy N. Molecular bases of progeroid syndromes. Hum Mol Genet 2006; 15 Spec No 2: R151-61.

[23] Fang XK, Gao Y, Yang HY, et al. Alleviating effects of active fraction of Euonymus alatus abundant in flavonoids on diabetic mice. Am J Chin Med 2008; 36: 125-40.

[24] Howlett HC, Bailey CJ. A risk-benefit assessment of metformin in type 2 diabetes mellitus. Drug Saf 1999; 20: 489-503.

[25] Salpeter SR, Greyber E, Pasternak GA, Salpeter EE. Risk of fatal and nonfatal lactic acidosis with metformin use in type 2 diabetes mellitus: systematic review and meta-analysis. Arch Intern Med 2003; 163: 2594-602.

[26] Farah IO. Differential modulation of intracellular energetics in A549 and MRC-5 cells. Biomed Sci Instrum 2007; 43: 110-5.

[27] Cha BY, Park CJ, Lee DG, et al. Inhibitory effect of methanol extract of Euonymus alatus on matrix metalloproteinase-9. J Ethnopharmacol 2003; 85: 163-7.

[28] Wood JP, Osborne NN. Zinc and energy requirements in induction of oxidative stress to retinal pigmented epithelial cells. Neurochem Res 2003; 28: 1525-33.

[29] Bressler NM, Bressler SB, Fine SL. Age-related macular degeneration. Surv Ophthalmol 1988; 32: 375-413.

[30] Friedman DS, O'Colmain BJ, Munoz B, et al. Prevalence of agerelated macular degeneration in the United States. Arch Ophthalmol 2004; 122: 564-72.

[31] Chang I, Cho N, Koh JY, Lee MS. Pyruvate inhibits zinc-mediated pancreatic islet cell death and diabetes. Diabetologia 2003; 46: 1220-7.

[32] Berry EV, Toms NJ. Pyruvate and oxaloacetate limit zinc-induced oxidative HT-22 neuronal cell injury. Neurotoxicology 2006; 27: 1043-51.

[33] Desagher S, Glowinski J, Premont J. Pyruvate protects neurons against hydrogen peroxide-induced toxicity. J Neurosci 1997; 17: 9060-7.

[34] O'Donnell-Tormey J, Nathan CF, Lanks K, DeBoer CJ, de la Harpe J. Secretion of pyruvate: an antioxidant defense of mammalian cells. J Exp Med 1987; 165: 500-14.

[35] Roberts E, Simonsen DG. Protection against hydrazine toxicity by alpha-ketoglutarate and oxalacetate: enhancement of arginine protection. Biochem Pharmacol 1965; 14: 351-3.

[36] Kuroda K, Akao M. Inhibitory effect of fumaric acid and dicarboxylic acids on gastric ulceration in rats. Arch Int Pharmacodyn Ther 1977; 226: 324-30.

[37] Willcox BJ, Curb JD, Rodriguez BL. Antioxidants in cardiovascular health and disease: key lessons from epidemiologic studies. Am J Cardiol 2008; 101: 75D-86D. (http://creativecommons.org/licenses/by-nc/3.0/) which permits unrestricted, non-commercial use, distribution and reproduction in any medium, provided the work is properly cited. 